\title{
Spatial coherence of thermal near fields
}

\author{
C. Henkel ${ }^{1}$ \\ Institut für Physik, Universität Potsdam, 14469 Potsdam, Germany \\ K. Joulain, R. Carminati, J.-J. Greffet \\ Laboratoire d'Energétique Moléculaire et Macroscopique, Combustion, Ecole \\ Centrale Paris, 92295 Châtenay-Malabry cedex, France ${ }^{2}$
}

\section{Abstract}

We analyze the spatial coherence of the electromagnetic field emitted by a half-space at temperature $T$ close to the interface. An asymptotic analysis allows to identify three different contributions to the cross-spectral density tensor in the near-field regime. It is shown that the coherence length can be either much larger or much shorter than the wavelength depending on the dominant contribution.

PACS numbers: 42.72 (black body radiation); 73.20.M (surface plasmons); 42.25.K

(coherence in wave optics); 07.79.F (scanning near field optical microscopy)

\section{Introduction}

The typical textbook example of an incoherent source is a thermal source. From the point of view of temporal coherence, its spectrum is 'given by Planck's function and modified by its emissivity. For usual sources, the emissivity is a smooth function of frequency. Thus, the spectral width is usually on the order of the peak frequency of Planck's function. From the point of view of spatial coherence, a thermal source is often assumed to be delta correlated. Yet, an exact form of the cross-spectral density tensor has been derived for a blackbody radiator and it has been shown that the spatial coherence length is $\lambda / 2[1]$. These exact results seem to support the statement that a thermal source is incoherent. Yet, one has to analyze more carefully the problem when dealing with a real thermal source. In this paper, we consider a source that consists of a half space filled with a lossy material at temperature $T$. We are interested in the emitted field so that we assume that there are no other sources. Thus there is no incident radiation illuminating the sample. Note in particular that this is not an equilibrium situation.

Since we explicitly introduce a model for the Carsten.Henkel@quantum.physik.uni-potsdam.de

2 Unité propre de recherche no. 288 du Centre National de la Recherche Scientifique 
source, the emitted field contains evanescent waves in the vicinity of the interface. These evanescent waves are not taken into account when dealing with blackbody radiation. Yet, they modify the coherence properties of the source in the near field as was shown in [2]. The effect is particularly striking if a resonant surface wave is excited. It has been shown that the coherence length can be either much larger than the wavelength or much shorter than $\lambda / 2$ close to the surface. Temporal coherence is also dramatically modified. For example, the emitted radiation is almost monochromatic when a surface wave is excited [3]. These results were obtained using a direct calculation of the field emitted by a half-space in the framework of fluctuation electrodynamics [4-7].

The subject of this paper is to analyze the spatial coherence of the emitted field by means of an asymptotic evaluation of the cross-spectral density tensor in the near-field limit (interfacedetector distance small compared to the wavelength). This analysis permits to retrieve the properties reported in [2] and yields insight into the physical mechanism responsible for these effects. We are thus able to identify all the possible contributions to the cross-spectral density tensor : thermally excited surface plasmons, skin-layer currents and small-scale polarization fluctuations. We show that to a good approximation, the sum of these three asymptotic contributions coincides with the exact result. We obtain different characteristic behaviours that vary in accordance with the dominant term. Surface waves such as surface plasmonpolaritons or surface phonon-polaritons yield long-range spatial coherence on a scale of the surface wave propagation length which may be much larger than the wavelength when aborption is small. On the contrary, skin-layer currents and small-scale polarization fluctuations lead to a much shorter spatial coherence length that only depends on the distance to the interface. A surprising consequence of this prop- erty is that the macroscopic theory of radiometry may be extended into the mesoscopic regime insofar as emission is concerned. Note however that this conclusion is based on the assumption of a local medium. The ultimately limiting scale is thus given by the electron screening length or the electron Fermi wavelength, whatever is larger $[8,9]$.

\section{Overview}

\subsection{Radiation emitted by a thermal source}

In this section, we review the source theory approach we use for the computation of the thermal electromagnetic field [4-7]. We focus on the radiation in the vacuum close to a source that we model as a linear dielectric with dielectric function $\varepsilon(\mathbf{r} ; \omega)$. The frequency dependence will not be indicated explicitly in the following since we calculate quantities at fixed frequency (or, equivalently, at fixed wavelength $\lambda=2 \pi c / \omega$. The source radiates because it contains a fluctuating polarization field $\mathbf{P}(\mathbf{r})$. The spectral density of this field is characterized by the crosscorrelation tensor $S_{P}^{i j}\left(\mathbf{r}_{1}, \mathbf{r}_{2}\right)$ that, according to the fluctuation-dissipation theorem $[4,5,10,11]$, is given by

$$
S_{P}^{i j}\left(\mathbf{r}_{1}, \mathbf{r}_{2}\right)=\frac{2 \hbar \varepsilon_{0} \operatorname{Im} \varepsilon\left(\mathbf{r}_{1}\right)}{\mathrm{e}^{\hbar \omega / k_{B} T}-1} \delta^{i j} \delta\left(\mathbf{r}_{1}-\mathbf{r}_{2}\right)
$$

The Kronecker $\delta^{i j}$ and the spatial $\delta$-function in this formula follow from the assumption that the dielectric function is isotropic and local. We have taken the normal-ordered form for the polarization field spectrum since we are ultimately interested in the electromagnetic field measured by a photodetector (given by normally-ordered field operators $[1,10])$. The electric field $\mathbf{E}(\mathbf{r})$ radiated by the polarization $\mathbf{P}(\mathbf{r})$ is now given by 
the Green function for the source geometry

$$
E_{i}(\mathbf{r})=\int_{V} \mathrm{~d} \mathbf{r}^{\prime} \sum_{j} G^{i j}\left(\mathbf{r}, \mathbf{r}^{\prime}\right) P_{j}\left(\mathbf{r}^{\prime}\right)
$$

where $V$ is the volume of the source, i.e., the domain where $\operatorname{Im} \varepsilon\left(\mathbf{r}^{\prime}\right)$ is nonzero according to (1). All quantities in (2) are understood as temporal Fourier transforms at frequency $\omega$. The coherence function $W^{i j}\left(\mathbf{r}_{1}, \mathbf{r}_{2}\right)$ of the electromagnetic field is now obtained as a thermal average of (2), using the polarization spectrum (1). One obtains $[2,3]$

$$
\begin{aligned}
W^{i j}\left(\mathbf{r}_{1}, \mathbf{r}_{2}\right)= & \frac{2 \hbar \varepsilon_{0}}{\mathrm{e}^{\hbar \omega / k_{B} T}-1} \sum_{k} \int_{V} \mathrm{~d} \mathbf{r}^{\prime} \operatorname{Im} \varepsilon\left(\mathbf{r}^{\prime}\right) \times \\
& \times G^{i k *}\left(\mathbf{r}_{1}, \mathbf{r}^{\prime}\right) G^{j k}\left(\mathbf{r}_{2}, \mathbf{r}^{\prime}\right)
\end{aligned}
$$

The problem is now to evaluate this expression analytically and to obtain an estimate for its dependence on the separation $\mathbf{s} \equiv \mathbf{r}_{2}-\mathbf{r}_{1}$ between the observation points.

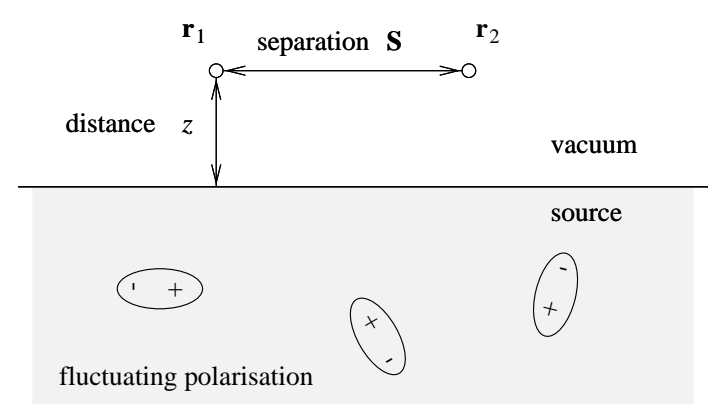

Fig.1: Model geometry for a planar source.

To proceed in the calculation, we focus on the simplified geometry shown in figure 1: an infinite half-space with uniform dielectric constant $\varepsilon$, separated by the plane $z=0$ from the empty half-space $z>0$. For this arrangement, the Green tensor is explicitly known as a spatial Fourier transform with respect to the lateral separation $\mathbf{S}=\left(s_{x}, s_{y}\right) \equiv \mathbf{R}_{2}-\mathbf{R}_{1}$. Details may be found in $[10,12,13]$ and in appendix A. As to be expected for this source geometry, the electric coherence tensor depends on the distances $z_{1}, z_{2}$ of the observers and their lateral separation $\mathbf{S}$. For simplicity, we put in the following $z_{1}=z_{2}=z$. We also normalize the coherence tensor $W^{i j}$ to its value for $\mathbf{r}_{1}=\mathbf{r}_{2}$ in the case of blackbody radiation

$$
W_{b b}=\frac{2 \hbar k^{3}}{3 \pi \varepsilon_{0}\left(\mathrm{e}^{\hbar \omega / k_{B} T}-1\right)}
$$

where as usual $k=\omega / c$. As outlined in appendix A, we thus get the following expression for the spatial Fourier transform of the coherence tensor

$$
\begin{aligned}
w^{i j}(\mathbf{S}, z) & =\frac{W^{i j}(\mathbf{S}, z)}{W_{b b}} \\
& =\int \frac{\mathrm{d}^{2} \mathbf{K}}{(2 \pi)^{2}} \mathrm{e}^{\mathrm{i} \mathbf{K} \cdot \mathbf{S}-2 z \operatorname{Im} \gamma} w^{i j}(\mathbf{K})
\end{aligned}
$$

where $\mathbf{K}$ denotes a wave vector parallel to the interface and $(\mathbf{K}, \gamma)$ is the vacuum wave vector of a plane wave emitted by the source. The tensor $w^{i j}(\mathbf{K})$ is given in appendix A, eq.(A.10). The integration over $\mathbf{K}$ in (5) also includes wave vectors $|\mathbf{K}|>k$, describing evanescent waves the source excites in the vicinity of the interface (the quantity $\gamma$ is then purely imaginary with positive imaginary part).

\subsection{Near field coherence function}

In this subsection, the typical behaviour of the field coherence function is discussed. We identify several distance regimes showing a very different behaviour of the lateral coherence function. Analytical approximations for the coherence function are deferred to the next section.

In figure 2 is shown the 'energy density' (the trace of the coherence tensor at coinciding posi- 

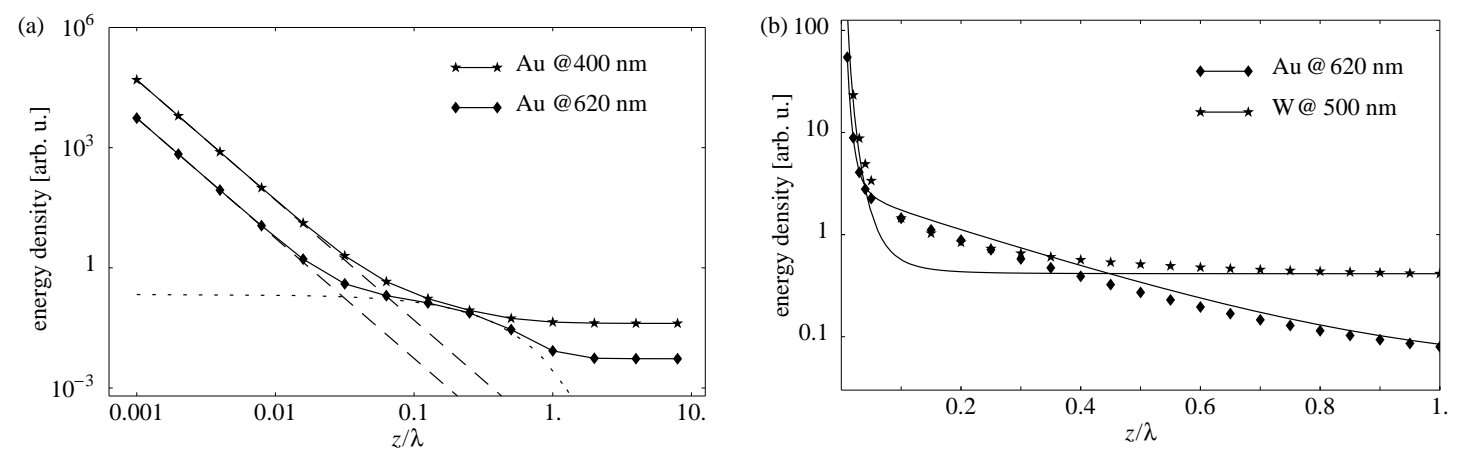

Fig. 2. Energy density $\operatorname{Tr} w^{i j}(\mathbf{S}=\mathbf{0}, z)$ vs. distance from a metal surface. Dots: numerical evaluation of the integral (5), solid lines: sum of the asymptotic approximations discussed in the text. In the far field, the numerically computed value is taken.

(a): $\log -\log$ scale for gold at $\lambda=620 \mathrm{~nm}(\varepsilon=-9.3+1.2 \mathrm{i})$ and at $\lambda=400 \mathrm{~nm}(\varepsilon=-1.1+6.5 \mathrm{i})$. The dielectric constants are extracted from [14]. Dashed line: $1 / z^{3}$ power law dominating the extreme near field; dotted line: exponentially decaying contribution of excited surface modes.

(b): log-linear scale, showing the exponentially decaying surface plasmon contribution for gold at $\lambda=620 \mathrm{~nm}$. For comparison, the case of tungsten at $\lambda=500 \mathrm{~nm}$ is shown where no plasmon resonance is found $(\varepsilon=4.4+18 \mathrm{i})$.

tions) above a metal surface in double logarithmic scale. One observes a strong increase with respect to the far field energy density when the distance $z$ is smaller than the wavelength. For moderate distances $z \leq \lambda$, the energy density is dominated by an exponentially increasing contribution [cf. fig.2(b)]. This is due to the excitation of surface plasmon resonances, whose contribution is calculated analytically in subsection 2.1. The other curve in fig.2(b) shows the energy density for the case of tungsten with $\operatorname{Re} \varepsilon>-1$ where no surface mode exists and no exponential increase is found. For small distances $z \ll \lambda$, the energy density follows a $1 / z^{3}$ power law ('static limit') that is discussed in subsection 2.2. The prefactor of this power law involves the imaginary part of the electrostatic reflection coefficient $\operatorname{Im}[(\varepsilon-1) /(\varepsilon+1)]$. The second curve in fig.2(a) illustrates the resonantly enhanced energy density for a wavelength where $\operatorname{Re} \varepsilon \approx-1$. The 'static limit' contribution then overwhelms that of the plasmon resonance.

In figure 3, we show the normalized lateral coherence function at chosen distances from the interface. In the far field [plot 3(a)], the coher- ence length is $\lambda / 2$, and the coherence function the same as for the blackbody field $((\sin k s) / k s$ behaviour). This is not surprising since at large distances $z \gg \lambda$, only propagating plane waves radiated into the vacuum half space contribute to the field.

When the surface plasmon excitation dominates the field energy $(z \leq \lambda)$, the field coherence extends over much longer distances [plot 3(b)]. This is because of the weak damping of the plasmon modes in this case. We show below (subsection 2.1) that the coherence length is indeed given by the plasmon propagation length. The figure also shows that the field is strongly polarized perpendicular to the interface, as is the surface plasmon mode.

At distances $z \ll \lambda$ even closer to the source, the field coherence length gets shorter again [plot 3(c)]. We show below that in this regime, the field behaves as if it was quasi-static (subsection 2.2). This leads to a lateral coherence length equal to the vertical distance $z$ from the interface and hence much shorter than the wave length. We thus find the surprising result that 
thermal near fields have no lower limit in their coherence length, as long as the dielectric function of the source may be taken as local.

One might finally ask whether the skin depth $\delta$ [defined in (23)] is relevant for the radiation emitted by a metallic source. This question is discussed in subsection 2.3 where we show that in the regime $\delta \ll z \ll \lambda$, a different power law $\left(\propto 1 / z^{2}\right)$ governs the energy density (see fig.4(a) below). The lateral coherence behaves similar to the static regime $z \ll \delta$, however, as shown in fig.4(b) below.

\section{Analytical approximations}

\subsection{Plasmon contribution}

It is well known that a dielectric-vacuum interface supports surface plasmon polariton (or phonon polariton) modes provided the dielectric constant satisfies $\operatorname{Re} \varepsilon<-1[15,16]$. These surface modes propagate parallel to the surface with a wave vector $K_{\mathrm{pl}}$ and are exponentially localized in the direction perpendicular to the interface. In addition, if there are losses in the dielectric, the propagation parallel to the interface is damped which may be described by a nonzero imaginary part of $K_{\mathrm{pl}}$. Mathematically, we obtain the plasmon dispersion relation by identifying the poles of the transmission coefficients $t_{\mu}(\mu=\mathrm{s}, \mathrm{p})$ as a function of the wave vector $K$. Only the p-polarization (magnetic field perpendicular to the plane of incidence) gives a pole at the (complex) position

$$
K_{\mathrm{pl}}=k \sqrt{\frac{-\varepsilon}{-\varepsilon-1}}, \quad \operatorname{Re} K_{\mathrm{pl}}, \operatorname{Im} K_{\mathrm{pl}}>0(6)
$$

The plasmon pole shows up as a sharp peak when the integrand $w^{i j}(\mathbf{K})$ in (5) is plotted as a function of the lateral wave vector magnitude $K$ [cf. eq.(A.10)]. This suggests that we get a good approximation to the plasmon contribution by taking slowly varying terms outside the integral and evaluating the pole contribution by contour integration. For example, the denominator of the $\left|t_{\mathrm{p}}\right|^{2}$ transmission coefficient may be approximated as

$$
\frac{1}{\left|\varepsilon \gamma+\gamma_{2}\right|^{2}} \approx \frac{4|\varepsilon|^{2}}{|\varepsilon+1||\varepsilon-1|^{2}} \operatorname{Im} \frac{1}{K^{2}-K_{\mathrm{pl}}^{2}}(7)
$$

where $\gamma=\gamma(K)$ and $\gamma_{2}$ are the vertical wave vector components above and below the interface. It is essential for the contour integration to work that one expresses the absolute square on the left hand side as the imaginary part of an analytic function of $K$ (right hand side).

It is easily checked from (A.10) that the trace of $w^{i j}(\mathbf{K})$ only depends on the magnitude $K$ of the lateral wave vector $\mathbf{K}$. The integration over the angle between $\mathbf{K}$ and $\mathbf{S}$ therefore gives

$$
\begin{aligned}
& \operatorname{Tr} w^{i j}(\mathbf{S}, z)= \\
& \int_{0}^{\infty} \frac{K \mathrm{~d} K}{2 \pi} J_{0}(K s) \mathrm{e}^{-2 z \operatorname{Im} \gamma} \operatorname{Tr} w^{i j}(\mathbf{K})
\end{aligned}
$$

where $J_{0}(\cdot)$ is the ordinary Bessel function and $s=|\mathbf{S}|$. The individual tensor components also involve Bessel functions $J_{2}(K s)$, as discussed in appendix B.1. The integration over $K$ may be done using the identity (B.2) proven in appendix B.2.1. The diagonal elements of the coherence tensor finally take the suggestive form

$$
\begin{aligned}
w^{i i}(\mathbf{S}, z) & \approx C_{\mathrm{pl}} \mathrm{e}^{-2 \kappa_{\mathrm{pl}} z} g^{i}\left(K_{\mathrm{pl}} s\right) \\
g^{\|}(u) & =\frac{1}{2} \operatorname{Re}\left[H_{0}(u)-H_{2}(u)-\frac{4 \mathrm{i}}{\pi u^{2}}\right] \\
g^{\perp}(u) & =\frac{1}{2} \operatorname{Re}\left[H_{0}(u)+H_{2}(u)+\frac{4 \mathrm{i}}{\pi u^{2}}\right] \\
g^{z}(u) & =|\varepsilon| \operatorname{Re} H_{0}(u) \\
C_{\mathrm{pl}} & =\frac{3 \pi}{2} \frac{|\varepsilon|^{2}}{|\varepsilon+1|^{7 / 2}} .
\end{aligned}
$$



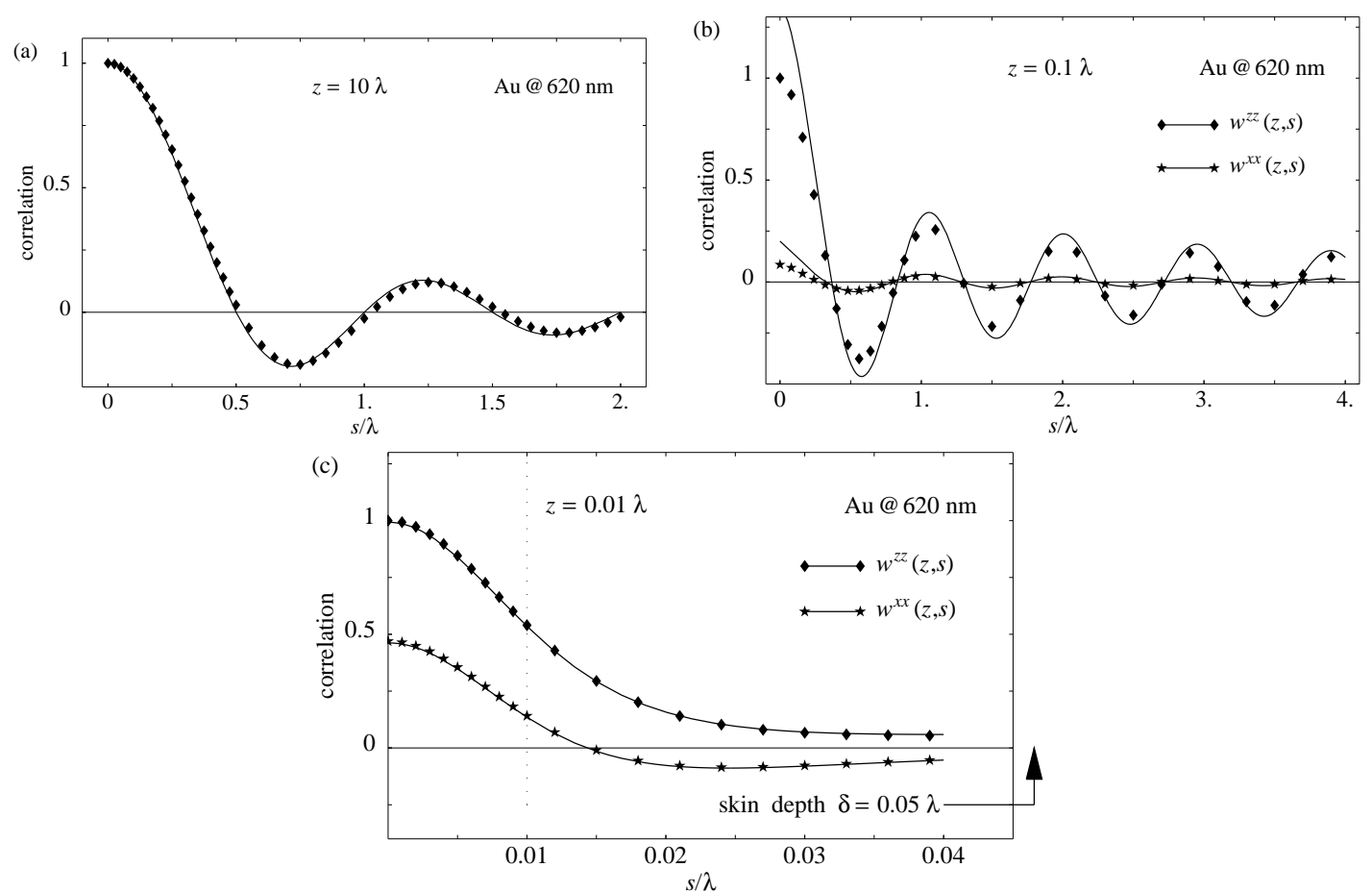

Fig. 3. Normalized lateral coherence functions for three fixed distances $z$, plotted vs. the lateral separation $s=|\mathbf{S}|$. All plots are for a gold surface at $\lambda=620 \mathrm{~nm}$. Dots: numerical evaluation of (5), solid lines: analytical approximations discussed in the text. The numerically computed values were used to normalize all curves.

(a): far field regime $z=10 \lambda$. The trace of the coherence tensor is plotted, normalized to its value for $s=0$. Solid line: free space coherence function $\sin (k s) /(k s)$.

(b): plasmon dominated regime $z=0.1 \lambda$. The components $w^{x x}$ and $w^{z z}$ are plotted, normalized to (the numerically computed) $w^{z z}(\mathbf{S}=\mathbf{0}, z)$.

(c) static regime $z=0.01 \lambda$. The components $w^{x x}$ and $w^{z z}$ are plotted and normalized as in plot $3(\mathrm{~b})$. The solid curve only contains the extreme near-field contribution (17).

where $\kappa_{\mathrm{pl}}=\operatorname{Im} \gamma\left(K_{\mathrm{pl}}\right)$ is the perpendicular plasmon decay constant and $H_{0,2}(\cdot) \equiv H_{0,2}^{(1)}(\cdot)$ are Hankel functions (or Bessel functions of the third kind) [17]. The superscripts $\|, \perp$ indicate the directions parallel and perpendicular to the separation vector $\mathbf{S}$ in the interface plane.

Eq.(9) shows that the plasmon resonance gives a contribution to the energy density that increases exponentially when approaching the source. This behaviour is reproduced by the numerical evaluation of (A.10), as shown in fig. 2(b). As a function of the lateral distance $s$, the correlation tensor eq.(9) shows damped oscillations whose wavelength is fixed by the plasmon wave vector
$K_{\mathrm{pl}}$, as shown in fig.3(b). These oscillations can be made explicit using the asymptotic form of the Hankel function [17]

$$
\begin{aligned}
& \left|K_{\mathrm{pl}} s\right| \gg 1: \\
& H_{n}\left(K_{\mathrm{pl}} s\right) \approx \sqrt{\frac{2}{\pi K_{\mathrm{pl}}} s} \mathrm{e}^{\mathrm{i}\left(K_{\mathrm{pl}} s-\pi / 4-n \pi / 2\right)}
\end{aligned}
$$

We thus conclude that the propagation distance of the plasmon resonance, as contained in the imaginary part of $K_{\mathrm{pl}}$, determines the coherence length of the field in this regime. For a dielectric constant with small imaginary part, the inverse 
propagation distance is approximately

$$
\operatorname{Im} K_{\mathrm{pl}} \approx \frac{\left(\operatorname{Re} K_{\mathrm{pl}}\right)^{3}}{k^{2}} \frac{\operatorname{Im} \varepsilon}{2(\operatorname{Re} \varepsilon)^{2}} \ll k .
$$

Thermally excited plasmons thus lead to a spatially coherent field on a length scale well exceeding the vacuum wave length. They also create a net field polarization, as shown by the anisotropy of the tensor elements in (10-12) [see also fig.3(b)]. This anisotropy may be understood from the fact that the coherence between points separated by $\mathbf{S}$ is created by plasmons propagating parallel to this direction, and the latter are polarized in the plane spanned by $\mathbf{S}$ and the normal vector $\mathbf{e}_{z}$.

\subsection{Extreme near field: quasi-static regime}

We now turn to the near field limit $z \ll \lambda$. Inspecting the integrand of (A.10), one finds that in addition to the plasmon resonance, large wave vectors $K \gg k$ dominate the integral. This is because the exponential cutoff $\mathrm{e}^{-2 z \operatorname{Im} \gamma(K)} \approx$ $\mathrm{e}^{-2 z K}$ gets effective only for $K \geq 1 / z \gg k$. We thus obtain the asymptotic behaviour of the integral when we expand the integrand to leading order in the limit $1 / z \geq K \gg k$. The transmission coefficients, e.g., become in this limit

$$
\begin{aligned}
\left|t_{\mathrm{p}}\right|^{2} & \approx \frac{4\left|\gamma_{2}\right|^{2}|\varepsilon|}{K^{2}|\varepsilon+1|^{2}}\left(1+\frac{k^{2}}{K^{2}} \operatorname{Re} \frac{\varepsilon}{\varepsilon+1}\right) \\
\left|t_{\mathrm{s}}\right|^{2} & \approx \frac{4\left|\gamma_{2}\right|^{2}}{K^{2}}\left(1+\frac{k^{2}}{4 K^{2}} \operatorname{Re}(\varepsilon+1)\right)
\end{aligned}
$$

We perform the integration over $K$ using eq.(B.8), as explained in appendix B.2.2 and get the following asymptotic form for the diagonal elements of the cross-correlation tensor

$$
w^{i i}(\mathbf{S}, z) \approx \frac{C_{\mathrm{xnf}}}{(k z)^{3}} g^{i}(s / z)
$$

$$
\begin{aligned}
g^{\|}(u) & =\frac{1-u^{2} / 2}{\left(1+u^{2} / 4\right)^{5 / 2}} \\
g^{\perp}(u) & =\frac{1}{\left(1+u^{2} / 4\right)^{3 / 2}} \\
g^{z}(u) & =\frac{2-u^{2} / 4}{\left(1+u^{2} / 4\right)^{5 / 2}} \\
C_{\mathrm{xnf}} & =\frac{3}{32} \operatorname{Im} \frac{\varepsilon-1}{\varepsilon+1}=\frac{3}{16} \frac{\operatorname{Im} \varepsilon}{|\varepsilon+1|^{2}}
\end{aligned}
$$

The coherence tensor given by (17) shows a power law increase $1 / z^{3}$ when the interface is approached, as plotted in fig. 2(a). It therefore takes over compared to the plasmon contribution in the 'extreme near field limit' $z \ll 1 / \kappa_{\text {pl }}$. In this regime, the lateral coherence of the field is characterized, as shown by the scale functions $g^{i}(s / z)$ in (18-20), by a lorentzian shape whose scale is set by the distance $z$ to the source. Hence, the closer one detects the field, the more it is spatially incoherent.

This behaviour may be understood from electrostatics: in the near field, the electromagnetic fields behave as if they were quasi-static because they vary on a length scale much smaller than the wave length (retardation is negligible). A near field detector is thus sensitive to a source area of the order of $\pi z^{2}$, and spatial coherence is observed when these areas overlap, hence for a separation smaller than the distance $z$. Similar arguments have also been put forward to interpret subwavelength resolution in optical near field microscopy $[18,19]$. The electrostatic analogy may be pushed even further: it is easily checked that we get the same result as (17) using electrostatic image theory. As a consequence of the fluctuation-dissipation theorem $[4,5,10,11]$, we have indeed

$$
\begin{aligned}
& W^{i j}\left(\mathbf{r}_{2}, \mathbf{r}_{1}\right) \propto \operatorname{Im} G^{i j}\left(\mathbf{r}_{2}, \mathbf{r}_{1}\right) \\
& \propto \operatorname{Im} E_{\text {image }}^{i}\left(\mathbf{r}_{2} ; \overline{\mathbf{r}}_{1}, \overline{\mathbf{d}}_{j}\right),
\end{aligned}
$$

where $G^{i j}$ is again the electric Green function. The electric field $\mathbf{E}_{\text {image }}$ is created by the image 
$\overline{\mathbf{d}}_{j}$ of a dipole $\mathbf{d}_{j}$ (polarized along the $x^{j}$-axis) at position $\mathbf{r}_{1}$, the image dipole being located at the mirror position $\overline{\mathbf{r}}_{1}=\left(x_{1}, y_{1},-z_{1}\right)$. This image dipole field dominates the Green function $G^{i j}$ at sufficiently close distance from the source if the electrostatic reflection coefficient $(\varepsilon-1) /(\varepsilon+1)$ has a nonzero imaginary part [see eq.(21)].

We stress that there is no lower limit to the spatial coherence length of the near field, provided one uses the framework of a local dielectric susceptibility. Model calculations for a free electron gas confined to a half-space show that this framework breaks down at wave vectors $K$ of the order of the Fermi wave vector $[8,9]$. For our problem, this corresponds to typical distances of the order of $0.1 \mathrm{~nm}$ that are difficult to achieve for near field probes even in the optical range.

\subsection{Relevance of the skin depth}

It has become clear from the two preceding subsections that the lateral coherence of near field radiation strongly depends on the distance of observation to the source. It might have been expected that the skin depth shows up in this discussion, since it governs the penetration depth for electric fields into the metal. We conclude our analytical work by identifying the relevance of this length scale.

Recall that the skin depth is given by

$$
\delta=\frac{\lambda / 2 \pi}{\operatorname{Im} \sqrt{\varepsilon}}
$$

For good conductors and low frequencies, the dielectric function is dominated by its zerofrequency pole

$$
\varepsilon=\frac{\mathrm{i} \sigma}{\varepsilon_{0} \omega}
$$

where $\sigma$ is the (possibly frequency dependent) conductivity. In particular, one has $|\varepsilon| \gg 1$ and $\delta \ll \lambda$ in this regime: the source material hence approaches a perfect conductor. This implies that the large $K$ expansion of the transmission coefficients in (A.10) has to be reconsidered: while the limit $K \gg k$ may be justified, the limit $K \gg k|\sqrt{\varepsilon}|$ may be not. We find that there exists an intermediate distance regime $\delta \ll z \ll \lambda$ (corresponding to wave vectors $k|\sqrt{\varepsilon}| \gg K \gg k$ ) where the coherence tensor shows a different behaviour [20]. The expansion of the transmission coefficients in this regime reads

$$
\begin{aligned}
\left|t_{\mathrm{p}}\right|^{2} & \approx \frac{4\left|\gamma_{2}\right|^{2}}{|\varepsilon| K^{2}}\left[1+\mathcal{O}\left(\frac{K}{k \sqrt{\varepsilon}}\right)\right] \\
\left|t_{\mathrm{s}}\right|^{2} & \approx \frac{4\left|\gamma_{2}\right|^{2}}{|\varepsilon| k^{2}}\left[1+\mathcal{O}\left(\frac{K}{k \sqrt{\varepsilon}}\right)\right]
\end{aligned}
$$

We finally get an isotropic coherence tensor

$$
\begin{aligned}
w^{i i}(\mathbf{S}, z) & \approx \frac{3}{16} \frac{\delta}{k z^{2}} g(s / z) \\
g(u) & =\frac{1}{\left(1+u^{2} / 4\right)^{3 / 2}}
\end{aligned}
$$

The skin layer dominated regime is thus characterized by a $1 / z^{2}$ power law for the energy density. As shown in figure 4(a), the skin depth $\delta$ separates this regime from the extreme near field where a different power law $1 / z^{3}$ prevails. We observe from fig.4(b) and (26) that the lateral coherence length is equal to the distance $z$ from the source, as in the extreme near field regime. This is not so surprising since the field propagation in the vacuum half space above the source is governed by the length scales $\lambda$ and $z$, whatever the smaller, while the skin depth is only relevant for the propagation inside the source. 

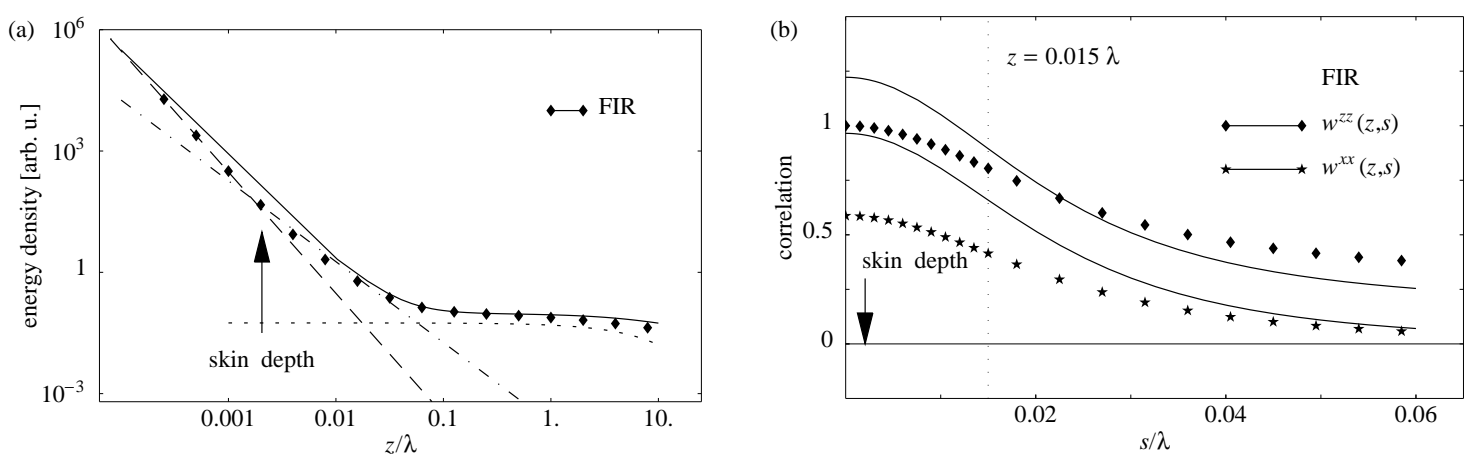

Fig. 4. Near field coherence in the skin-effect dominated regime $\delta \ll z \ll \lambda$, typical for metals in the far infrared. Dots: numerical evaluation of the integral (5), lines: analytic asymptotics discussed in the text. (a): energy density $\operatorname{Tr} w^{i j}(\mathbf{S}=\mathbf{0}, z)$ above a metallic surface with $\varepsilon=-8.26+\mathrm{i} 10^{4}$ (gold extrapolated to $\lambda=3.7 \mu \mathrm{m}$ ). The solid line is the sum of the asymptotic contributions derived in this paper. (b): normalized lateral coherence functions $w^{i i}(\mathbf{S}, z) / w^{z z}(\mathbf{0}, z)(i=x, z)$ for fixed distance $z$, plotted vs. the lateral separation $s=|\mathbf{S}|$. The $x$ - and $z$-polarizations differ because the plasmon contribution already comes into play. The numerically computed $w^{z z}(\mathbf{0}, z)$ was used to normalize all curves, this is why the analytic correlations exceed unity.

To conclude, we recall that the different contributions to the correlation tensor originate in distinct domains on the $K$-axis in the integral (5). The total correlation tensor is therefore given by the sum of the surface plasmon, extreme near field, and skin-layer contributions. The accuracy of this approximation is visible in figs.2, 4(a). Note that in the figures, the numerically computed far-field energy density has been added to get the correct large distance limit.

\section{Conclusion}

In the near field, the spatial coherence of thermal radiation differs strongly from the blackbody field. Confined field modes like surface plasmon polaritons that propagate along the source surface make the field spatially coherent over large scales if they dominate the radiation density. At close distance (smaller than the skin depth), the radiation is dominated by quasistatic fields, and the coherence length drops well below the wave length, being limited only by the (non)locality of the dielectric response of the source material. The cross over between these regimes is determined by the skin depth and the electrostatic reflection coefficient. We conclude that in the near field, macroscopic concepts like a local emissivity are still meaningful at the subwavelength scale, provided coherent surface excitations are absent or subdominant.

The asymptotic forms for the cross spectral density tensor obtained in this paper are useful to characterize thermal noise fields that may perturb particles in integrated microtraps 'mounted' with electromagnetic fields above a solid surface [21-28]. The concomitant scattering and decoherence of the guided matter waves is discussed elsewhere $[29,30]$.

The fluctuation electrodynamics used in this paper enabled us to treat a non-equilibrium situation (thermal source in vacuum at $T=0$ ) where the fluctuation-dissipation theorem for the electric field is not immediately applicable. In particular, we neglected the zero-point radiation impinging on the interface from the empty halfspace. The domain of validity of this approximation, as well as the calculation of anti-normal ordered correlation functions will be the subject of future investigations. 
3.0.0.1 Acknowledgements. C.H. thanks S. Scheel for many fruitful exchanges.

\section{A Notations for the plane interface}

The Green tensor describing the emission from the source $z^{\prime}<0$ into the vacuum half space $z>0$ may be written in spatial Fourier space as $[10,12]$

$$
\begin{aligned}
& G^{i j}\left(\mathbf{r}, \mathbf{r}^{\prime}\right)=\int \frac{\mathrm{d}^{2} \mathbf{K}}{(2 \pi)^{2}} \mathrm{e}^{\mathrm{i} \mathbf{K} \cdot\left(\mathbf{R}-\mathbf{R}^{\prime}\right)} G^{i j}\left(\mathbf{K}, z, z^{\prime}\right) \\
& G^{i j}\left(\mathbf{K}, z, z^{\prime}\right)=\frac{\mathrm{i} k^{2}}{2 \varepsilon_{0} \gamma_{2}} \\
& \times \sum_{\mu=\mathrm{s}, \mathrm{p}} e_{\mu, i}^{(t)} e_{\mu, j}^{(2)} t_{\mu} \mathrm{e}^{\mathrm{i}\left(\gamma z-\gamma_{2} z^{\prime}\right)}
\end{aligned}
$$

We use bold capitals to denote vectors parallel to the interface, e.g., $\mathbf{K}=\left(k_{x}, k_{y}, 0\right)$. The vertical components of the wave vectors in vacuum and inside the source are, respectively,

$$
\begin{gathered}
\gamma=+\sqrt{k^{2}-\mathbf{K}^{2}}, \quad \operatorname{Im} \gamma>0 \\
\gamma_{2}=+\sqrt{\varepsilon k^{2}-\mathbf{K}^{2}}, \quad \operatorname{Im} \gamma_{2}>0
\end{gathered}
$$

The polarization vectors for the s- (or TE-) and p- (TM-)polarized waves are taken as

$$
\begin{aligned}
\mathbf{e}_{\mathrm{s}}^{(t)} & =\mathbf{e}_{\mathrm{s}}^{(2)}=\hat{\mathbf{K}} \times \hat{\mathbf{e}}_{z} \\
\mathbf{e}_{\mathrm{p}}^{(t)} & =\frac{K \hat{\mathbf{z}}-\gamma \hat{\mathbf{K}}}{k} \\
\mathbf{e}_{\mathrm{p}}^{(2)} & =\frac{K \hat{\mathbf{z}}-\gamma_{2} \hat{\mathbf{K}}}{\sqrt{\varepsilon} k}
\end{aligned}
$$

where $\hat{\mathbf{K}}$ is the unit vector parallel to K. Finally, with this choice for the polarization vectors, the Fresnel transmission coefficients are

$$
t_{\mathrm{s}}=\frac{2 \gamma_{2}}{\gamma+\gamma_{2}}, \quad t_{\mathrm{p}}=\frac{2 \gamma_{2} \sqrt{\varepsilon}}{\varepsilon \gamma+\gamma_{2}}
$$

When the Green tensor (A.1) is inserted into the integral (3), the spatial integration over $\mathbf{R}^{\prime}$ yields a $\delta$-function for the lateral wave vectors. The integration over $z^{\prime}$ is then

$$
\int_{-\infty}^{0} \mathrm{~d} z^{\prime} \mathrm{e}^{-\mathrm{i}\left(\gamma_{2}-\gamma_{2}^{*}\right) z^{\prime}}=\frac{1}{2 \operatorname{Im} \gamma_{2}}
$$

where the convergence is ensured by the positive imaginary part of $\gamma_{2}$. The resulting coherence tensor is then of the form (5). We use the identity

$$
k^{2} \operatorname{Im} \varepsilon=2 \operatorname{Im} \gamma_{2} \operatorname{Re} \gamma_{2}
$$

and get after some elementary algebra:

$$
\begin{aligned}
w^{i j}(\mathbf{K})= & \frac{3 \pi}{4 k} \frac{\operatorname{Re} \gamma_{2}}{\left|\gamma_{2}\right|^{2}} \times \\
& \times \sum_{\mu} e_{\mu, i}^{(t)} e_{\mu, j}^{(t) *}\left|\mathbf{e}_{\mu}^{(2)}\right|^{2}\left|t_{\mu}\right|^{2}
\end{aligned}
$$

\section{B Components of the coherence tensor}

In this appendix, we outline the calculation for the components of the coherence tensor.

\section{B.1 Angular integrations}

The only quantities in (A.10) that depend on the angle $\varphi$ between the lateral wavevector $\mathbf{K}$ and the separation $\mathbf{S}$ are the polarization vectors $\mathbf{e}_{\mu}$. To simplify the calculation, we choose the $x$-axis parallel to $\mathbf{S}$. We then get the following azimuthal integrals (eq.9.1.18 of [17])

$$
\int_{-\pi}^{\pi} \frac{\mathrm{d} \varphi}{\pi} \mathrm{e}^{\mathrm{i} K s \cos \varphi}\left(\begin{array}{c}
\sin ^{2} \varphi \\
\cos ^{2} \varphi
\end{array}\right)=J_{0}(K s) \pm J_{2}(K s)(\text { B. } 1)
$$


The integrals with $\sin \varphi \cos \varphi$ vanish due to parity. We also note that one also gets nonzero offdiagonal elements $W^{x z}, W^{z x}$ due to p-polarized modes. For simplicity, these are not discussed here.

\section{B.2 Radial integrations}

We are left with integrals over the radial wave vector $K$. These are worked out using the definitions (A.6) of the polarization vectors and the transmission coefficients (A.7).

\section{B.2.1 Plasmon pole}

To find the plasmon contribution, we extract, as mentioned in the main text, the pole of the $t_{\mathrm{p}}$ coefficient and approximate the other factors by their values at the pole. The remaining integral can be reduced to the following standard form

$$
\int_{0}^{\infty} \frac{x \mathrm{~d} x}{x^{2}-q^{2}} J_{0}(x s)=\frac{\mathrm{i} \pi}{2} H_{0}^{(1)}(q s)
$$

for $\operatorname{Im} q>0, s>0$. To prove this identity, we use contour integration. The Bessel function is written as ([17], eqs. 9.1.3, 9.1.39)

$$
J_{0}(x)=\frac{1}{2}\left[H_{0}^{(1)}(x)-H_{0}^{(1)}\left(\mathrm{e}^{\mathrm{i} \pi} x\right)\right]
$$

where $H_{0}^{(1)}(x)$ is the Hankel function. The integral may now be written as

$$
\frac{1}{2} \int_{\mathrm{e}^{\mathrm{i} \pi} \infty}^{\infty} \frac{x \mathrm{~d} x}{x^{2}-q^{2}} H_{0}^{(1)}(x s)
$$

with an integration path running just above the negative real axis. The Hankel function is analytic in the upper half plane and vanishes expo- nentially for $|x| \rightarrow \infty$ there [see eq.(14)]. Therefore, closing the integration contour with a half circle, the integral is given by the residue at the pole $x=+q$ (because $\operatorname{Im} q>0$ ), and we get

$$
\int_{\mathrm{e}^{\mathrm{i} \pi} \infty}^{\infty} \frac{x \mathrm{~d} x}{x^{2}-q^{2}} H_{0}^{(1)}(x s)=\mathrm{i} \pi H_{0}^{(1)}(q s)
$$

This proves (B.2). Taking the imaginary part, we find both the trace and the $z z$ component (12) of the correlation tensor (9).

For the $x x$ - and $y y$ components of the coherence tensor, we also need the integral (B.2) with the Bessel function $J_{2}(x s)$ instead of $J_{0}(x s)$ [cf. eq.(B.1)]. Using the same reasoning as above, this integral is transformed into

$$
\frac{1}{2} \int_{\mathrm{e}^{\mathrm{i} \pi} \infty}^{\infty} \frac{x \mathrm{~d} x}{x^{2}-q^{2}} H_{2}^{(1)}(x s)
$$

In addition to the pole at $x=q$, we now have a contribution from the $-4 \mathrm{i} /(x s)^{2}$ singularity of the Bessel $H_{2}^{(1)}(x s)$ function at the origin. This singularity lies on the integration path and is therefore taken into account by half its (negative) residue at $x=0$. Combining the latter with the residue at $x=q$, we get

$$
\int_{\mathrm{e}^{\mathrm{i} \pi} \infty}^{\infty} \frac{x \mathrm{~d} x}{x^{2}-q^{2}} H_{2}^{(1)}(x s)=\mathrm{i} \pi H_{2}^{(1)}(q s)-\frac{4 \pi}{q^{2} s^{2}}(\mathrm{~B} .7)
$$

We may verify the sign of the second term by checking that the function (B.7) vanishes in the limit $s \rightarrow 0$, as is the case for the left hand side of (B.6).

\section{B.2.2 Near field regime}

In the near field regimes $K \gg k|\sqrt{\varepsilon}|$ (extreme near field) and $k|\sqrt{\varepsilon}| \gg K$ (skin layer dominated regime), the expansions $(16,25)$ of 
the transmission coefficients are straightforward to obtain. The final integration involves integer powers of $K$ times products of Bessel functions and exponentials and is performed using the following identity

$$
\int_{0}^{\infty} \mathrm{d} K J_{0}(K s) \mathrm{e}^{-2 K z}=\frac{1}{\sqrt{4 z^{2}+s^{2}}}
$$

This may be proven starting from the Fourier expansion of the Coulomb potential (writing $\left.\mathbf{k}=\left(\mathbf{K}, k^{z}\right)\right)$

$$
\frac{1}{r}=\frac{1}{2 \pi^{2}} \int \mathrm{d}^{2} \mathbf{K} \mathrm{d} k^{z} \frac{\mathrm{e}^{\mathrm{ik} \cdot \mathbf{r}}}{\mathbf{k}^{2}}
$$

and evaluating the integral over the vertical wave vector component $k^{z}$ with contour integration (for $z>0$, a single pole at $k^{z}=\mathrm{i}|\mathbf{K}|$ contributes). The derivatives of (B.8) with respect to $z$ and $s$ then provide all necessary integrals.

\section{References}

[1] L. Mandel and E. Wolf, Optical coherence and quantum optics (Cambridge University Press, Cambridge, 1995).

[2] R. Carminati and J.-J. Greffet, Phys. Rev. Lett. 82 (1999) 1660.

[3] A. V. Shchegrov, K. Joulain, R. Carminati, and J.-J. Greffet, Phys. Rev. Lett. (2000), in press.

[4] E. M. Lifshitz, Soviet Phys. JETP 2 (1956) 73, [J. Exper. Theoret. Phys. USSR 29, 94 (1955)].

[5] D. Polder and M. V. Hove, Phys. Rev. B 4 (1971) 3303.

[6] J. Schwinger, J. Lester L. DeRaad, and K. A. Milton, Ann. Phys. (N.Y.) 115 (1978) 1.

[7] S. M. Rytov, Y. A. Kravtsov, and V. I. Tatarskii, Principles of Statistical Radiophysics (Springer, Berlin, 1989), Vol. 3, Chap. 3.
[8] P. J. Feibelman, Progr. Surf. Sci. 12 (1982) 287.

[9] G. W. Ford and W. H. Weber, Phys. Rep. 113 (1984) 195.

[10] G. S. Agarwal, Phys. Rev. A 11 (1975) 230.

[11] S. Scheel, L. Knöll, and D.-G. Welsch, Phys. Rev. A 58 (1998) 700.

[12] A. A. Maradudin and D. L. Mills, Phys. Rev. B 11 (1975) 1392.

[13] C. Henkel and J.-Y. Courtois, Eur. Phys. J. D 3 (1998) 129.

[14] E. Palik, Handbook of optical constants of solids (Academic, San Diego, 1985).

[15] Surface Polaritons, edited by V. M. Agranovich and D. L. Mills (North-Holland, Amsterdam, 1982).

[16] H. Raether, Surface Plasmons on Smooth and Rough Surfaces and on Gratings, Vol. 111 of Springer Tracts in Modern Physics (Springer, Berlin, Heidelberg, 1988).

[17] Handbook of Mathematical Functions, ninth ed., edited by M. Abramowitz and I. A. Stegun (Dover Publications, Inc., New York, 1972).

[18] J.-J. Greffet and R. Carminati, Progr. Surf. Sci. 56 (1997) 133.

[19] C. Henkel and V. Sandoghdar, Opt. Commun. 158 (1998) 250.

[20] C. Henkel, S. Pötting, and M. Wilkens, Appl. Phys. B 69 (1999) 379.

[21] J. P. Dowling and J. Gea-Banacloche, in Adv. At. Mol. Opt. Phys., edited by P. R. Berman (Academic Press, New York, 1997), Vol. 37, Suppl. 3, pp. 1-94.

[22] V. I. Balykin, in Adv. At. Mol. Opt. Phys., edited by B. Bederson and $\mathrm{H}$. Walther (Academic, San Diego, 1999), Vol. 41, pp. 181260.

[23] J. Schmiedmayer, Eur. Phys. J. D 4 (1998) 57. 
[24] M. Key et al., Phys. Rev. Lett. 84 (2000) 1371.

[25] J. Reichel, W. Hänsel, and T. W. Hänsch, Phys. Rev. Lett. 83 (1999) 3398.

[26] R. Folman, P. Krüger, D. Cassettari, B. Hessmo, T. Maier, and J. Schmiedmayer, Phys. Rev. Lett. 84 (2000) 4749.

[27] N. H. Dekker et al., Phys. Rev. Lett. 84 (2000) 1124.
[28] D. Müller, D. Z. Anderson, R. J. Grow, P. D. D. Schwindt, and E. A. Cornell, Phys. Rev. Lett. 83 (1999) 5194.

[29] C. Henkel and M. Wilkens, acta phys. slov. 50 (2000) 293 quant-ph/0005038.

[30] C. Henkel and S. Pötting, "Coherent transport of matter waves", submitted for publication in Appl. Phys. B. quant-ph/0007083 